\title{
2 The role of openness in the economic growth of regions
}

\subsection{The perspective of economic growth theory}

The extent of openness understood as the level of integration with the global economy is one of the so-called deep determinants of economic growth. The nexus between openness and economic growth is complex, and the issue gets even more complicated if we acknowledge the existence of heterogeneous regions within the body of the nation-state. The flows of goods, services as well as production factors or technology thus happens within a given region (intraregional), between regions of a given state (interregional) and last but not least internationally. The last dimension is obviously of principal significance.

The traditional growth theory is applied to the level of nations. However, regions can be treated as SOEs. In his seminal paper, North (1955) pointed to "A fundamental difficulty ... that the theory of regional economic growth had little relevance for the development of regions". A spatial approach of the main branch of models being a major disadvantage. At the same time, authors pointed that for instance the concept of the regional export base has significance but is only one of the large number of determinants of regional economic growth that should be investigated (Tiebout, 1956).

The emergence of NEG models, especially of dynamic type (incorporating the evolution of the economic system over time and space), allowed some progress to be made - in particular incorporating the spatial perspective; however, in highly simplified systems. The most recent development is the inclusion of firm heterogeneity.

\subsection{The role of openness in economic growth theory}

The nexus between openness and economic growth is one of the most important areas of academic analysis both on the theoretical and empirical front. The issue has been and still is addressed by various fields of inquiry such as classical and new growth theory, international economics or economic geography, and finally NEG.

In the neoclassical growth theory (Solow, 1956; Swan, 1956) openness does not matter in the long-run as growth is independent of economic policy. It can only lead to the so-called level effect just affecting the level of the real GDP per capita in the steady-state and not the growth rate. In the short-run, capital deepening is the major source of growth as income per capita is directly proportional to the level of capital per capita. The level of real GDP per capita in the steady-state is a positive function of 
the rate of saving (and thus investment rate) and a negative function of the population growth rate and depreciation of capital. The technological progress of exogenous character affects the level positively. The only factor affecting the long-run growth rate is the rate of exogenous technological progress. Namely, it is equal to the rate of technological progress. In this setting, the impact of an increase in the extent of openness due, for instance, to changes in trade policy on economic growth is only temporary. Neoclassical growth theory predicts, furthermore, that regions will converge in the long run under the assumption of perfect competition (absolute $\beta$ convergence or the catching-up level).

In an augmented model of Mankiw, Romer, and Weil (1992), human capital accumulation and the human capital endowment are considered, additionally. The model can better explain the observed variation in the level of economic development. It does not, however, modify the prior conclusions. The augmented neoclassical model by Brodzicki (2015) takes further the impact of infrastructure into account. In the model, following Mincerian tradition, the average level of education may be specified as a function of average years of schooling and average years of experience Bils and Klenow (2000).

Camacho, Carmen, and Zou (2004) proposed a spatial version of the Solow model in continuous time and space. With a standard neoclassical production function, the steady-state exists, and convergence gradually takes place. If one assumes homogeneity of space, then at the steady-state, all locations have the same level of physical capital. However, if spatial heterogeneity is introduced at the level of the technology or savings rate, regional differences persist, and there is no convergence.

The emergence of the endogenous growth and new trade theories (Aghion and Howitt, 1990, 1998; Lucas, 1988; Romer, 1986, 1990) has led to the reopening of the debate on the role of trade and more general the degree of openness in determining economic growth in the medium and long-term. The models of the first and second generation endogenised the rate of growth of technology either by allowing for the impact of human capital or introducing a separate R\&D sector purposefully producing knowledge in the form of patents. It is worth pointing out, however, that even in a semi-endogenous model of Ben-David and Loewy (2002), openness to trade through its impact on the process of accumulation of knowledge and technology transfer (knowledge diffusion) leads to endogenisation of the economic growth process.

The new growth theory models of Rivera-Batiz and Romer (1991) or Grossman and Helpman (1995) lead to different policy conclusions. A policy shift leading to a greater extent of openness (such as trade liberalisation), creates a permanent effect - the long-run growth rate is affected but not only positively, an adverse impact is also possible. In brief, the balance of costs and benefits of greater openness (liberalisation) depends on the nature and the exact product structure of trade - in other words, it's not merely related to the intensity of trade but also its composition (what exactly a given region produces, exports and imports). 
Greater openness to trade affects the rate of accumulation of knowledge, mostly through imports. They work as a channel allowing absorption of more advanced knowledge positively affecting the overall efficiency and thus the growth rate. RiveraBatiz and Romer (1991) show, however, that whether the effect is positive or adverse depends on the distance of economy from the global technology frontier (GTF) and the nature of diffusion of knowledge (perfect versus imperfect). Imperfect knowledge flows, coupled with openness can harm underdeveloped states or lagging regions.

Barro and Sala-i-Martin (2004) or Aghion and Howitt (2009) emphasize the role of technology diffusion in both absolute (the catching up effect) and conditional beta-convergence. It is mostly because imitation and implementation of innovation are cheaper than initial innovation. Imitation and adaptation still entail substantial costs which can be however lowered through more intense trade (mostly imports) or superior human capital and skills base. Nelson and Phelps (1966) stress that followers tend to grow faster; the greater is the initial gap from the leader. The gap diminishes over time, and thus the followers' growth rates tend to decrease alongside. In the steady-state, the leader and the follower grow at the same rate. Nelson and Phelps (1966) argue that education could positively affect the speed of adoption of new technologies. They distinguished the theoretical (potential) level of knowledge from the prevailing (existing) level of technology.

Benhabib and Spiegel (1994) extended the original model by adding an extra innovation term that controls for the impact of own capacity to develop knowledge on top of the ability to absorb external knowledge. Abreu et al. (2004) further accounted for potential spatial dependencies between bordering economies. Ciołek and Brodzicki (2017) in their empirical model, extend it even further by including two potential channels for technology diffusion through imports and FDI inflows and testing it for Polish regions. The two aforementioned channels find support in many empirical studies.

In their seminal study, Coe and Helpman (1995) studied the impact of trade on technology diffusion and found that international R\&D spillovers were related to imports and in particular to the composition of imports. Furthermore, a strong correlation between R\&D embodied in (bilateral) trade flows and total factor productivity (TFP) growth was identified. Coe, Helpman, and Hoffmaister (1997) endorsed the impact of domestic and foreign R\&D capital stocks on the TFP even after controlling for human capital. They extended the analysis by the inclusion of institutional variables, allowing for parameter heterogeneity based on institutional characteristics. The results suggested that institutional differences were significant determinants of TFP, and they had an impact on the degree of R\&D spillovers. In the context of the second channel considered, Hejazji and Safarian (1999) identified significant R\&D spillovers through FDI from the largest industrial countries to smaller OECD Member States. Xu (2000), in contrast, found that the technology transfer of US multinationals contributed to productivity growth but only in the group of developed economies. 
According to Keller (2002), for most countries, foreign sources of technology are crucial (they account for $90 \%$ or more) in productivity growth. They are, at the same time, more valuable for small and relatively poorer countries. It could be related to the significance of the variation in domestic R\&D investments. There is no indication that the process of international diffusion, and thus learning is inevitable, simple, or automatic. Imports are the primary channel of international technology diffusion with no indication of learning-by-exporting effects. FDI effects are, however, present, but the impact is highly asymmetric. Keller (2004) points out that technological knowledge spillovers appear to be resulting from a deliberate commitment to learning and matching international performance standards through ongoing interactions with foreigners. At the same time, local efforts seem to be necessary for successful technology adoption.

It could, therefore, mean that technology diffusion is not only spatially bounded but also not-universal. The follower must have a minimum level of endowments in order to be able to absorb technology. Technological change can thus be skill-based which could lead to technology-skill mismatch and thus non-convergence in the TFP levels (Acemoglu and Zilibotti, 2001).

Eaton and Kortum (2001) constructed in turn a model of innovation, growth, and trade with technology spillovers which pointed to convergence in income levels. The benefits of the larger market can be exploited by an innovator through exports. However, the innovator, in an open economy, must compete not only with domestic rivals but also with imported technologies. These are two offsetting forces. If they offset completely, only static gains from trade arise with no dynamic gains through technology accumulation. Decreased barriers to trade stimulate research activity characterized by the presence of scale effects. Real wages depend on the productivity of workers but also the size of the population. In the extreme case of autarky, relative real wages in the model depend on relative labour forces weighted by research productivity. Decreasing barriers to trade, however, benefit smaller economies to a large extent. On the other extreme, with zero gravity (a costless trade), relative real wages depend solely on relative research productivity with the size of the economy playing no significant role.

In an extension, Eaton and Kortum (2002), construct a Ricardian model accounting for realistic geographic features where bilateral trade is a function of absolute advantages, trade-promoting comparative advantages, and trade-resisting geographic barriers. The concept of trade gravity is thus fully integrated and accounted for.

Howitt (2000) in a multi-country endogenous Schumpeterian growth model, shows that due to technology diffusion, only R\&D-performing countries grow in the long run and converge to similar growth paths, while non-R\&D-performing countries stagnate. In this framework, an increase in the investment rate or the R\&D-subsidy rate in any R\&D-producing country can increase the overall growth rate. In an extension, Aghion, Howitt, and Mayer-Foulkes (2005) attribute the emergence of convergence clubs in income to R\&D potential and knowledge diffusion. In their stylized 
model, countries sort themselves into three groups: members of the highest group converge to a steady-state where they perform leading-edge R\&D (at the GTF), while the intermediate group converges to a steady-state where they only implement technologies developed elsewhere. High and intermediate group countries share the same growth rates in the long run as a result of technology diffusion; nonetheless, inequality between them in terms of development levels, increases. Economies of the lowest group grow at a slower rate and are unable to converge due to their inability to absorb knowledge from the GTF. In this set-up, the initial distance to the technological frontier matters and countries lagging by a significant distance can be entrapped. This model can be easily adapted to the regional context with innovative regions, catchingup regions, and lagging or falling behind regions.

It is also worth addressing the direction of causality between openness and economic growth. If openness affects growth than we deal with the so-called export-led growth (ELG) process through the channels described above. The term was introduced by Balassa (1978) and later investigated, among others by Marin (1992). Some theoretical work was conducted on the related concept of learning-by-exporting (Grossman and Helpman, 1995; Krugman, 1980b) or more recently at the firm level (Helpman, Melitz, and Yeaple, 2003; Melitz, 2003). The initial evidence was weak, and the general agreement to the existence of ELG is sometimes challenged (Dreger and Herzer, 2013).

On the other hand, the causality could be just the opposite - going from the growth to greater openness. Higher productivity in the larger domestic market (home marker effect, HME) could lead to greater international competitiveness and a subsequent increase in regional exports. At the same time, the demand for imports increases in the size of the regional economy. Thus, a bidirectional relationship is likely to exist if the processes described above hold simultaneously (Liu, Song, and Romilly, 1997).

It is worth stressing that in the new approach, Rodrik (2002) perceives openness or as he puts it the extent of integration as one of three deep fundamental determinants of economic growth alongside the quality of institutions and geographical conditions. Openness is treated here as a semi-endogenous factor shaped by purely exogenous geographical conditions (in particular of the first nature of geography) as well as institutional factors. Nonetheless, it, directly and indirectly, affects the shallow determinants of growth - related to the endowment of basic factors of production and the process of their accumulation as well as the overall productivity of the economic system.

Summing up, the initial literature review, from a theoretical standpoint, openness affects growth through several channels:

(a) first of all, it leads to the reallocation of factors of production to more productive sectors and thus to specialisation in accordance with the comparative or competitive advantage thus resources are allocated efficiently; 
(b) it leads to increased diffusion and accelerated absorption of knowledge and technology (technology transfer) in particular through imports (Coe and Helpman, 1995) or inflow of FDI (Branstetter, 2006);

(c) it stimulates the rate of innovation as it is frequently associated with an increase in the expenditures on $R \& D$;

(d) it allows better utilisation of scale economies and agglomeration externalities as a result of greater specialisation. At the same time, it leads to enhanced accumulation of factors of production;

(e) it stimulates competition in national and international markets, thus forcing companies to be more innovative.

\subsection{The perspective of NEG}

Further insight into the nexus linking openness to growth can be brought by the NEG literature. The NEG brought the issue of space into the mainstream economic theory. The seminal paper by Krugman (1991a) is generally regarded as the foundation of the NEG. It is an extension of the standard new trade theory model (Krugman, 1980a), allowing for interregional mobility of factors of production on top of the trade in final goods. Breinlich, Ottaviano, and Temple (2014) stress the fact that NEG theory is based on trade theory. Thus the relationship between external trade, internal economic geography, and regional disparities, is at its core. Fujita, Krugman, and Venables (2001) suggest that openness could work to disperse the manufacturing industry as a whole, but also lead to the spatial clustering of specific industries. External trade thus affects spatial patterns of activity and thus of trade by changing market access considerations (Hanson, 1996).

Incorporation of space into the theoretical framework and at the same endogenization of location choices requires moving beyond the neoclassical paradigm. It is crucial to reject the assumptions of the zero transport costs and allow for the presence of increasing returns to scale (both internal and external to a firm (plant)).

From a modelling point of view, NEG theories can be considered as an extension of new trade models allowing for simultaneous flows of goods as well as factors of production (capital and/or labour) and knowledge flows.

Fujita and Thisse (2004) state that there are three important assumptions in spatial modelling NEG:

(a) space is heterogeneous, which leads to the presence of comparative advantages in technology, natural resources, facilities or the presence of transport hubs or markets;

(b) externalities exist in both production and consumption - agglomeration forces arise from the bottom-up as a result of the non-market interactions between market agents - enterprises and households; classic Marshallian effects (Marshall, 1890; Marshall and Marshall, 1879) arise and specialisation 
related to the snowball effect in the event of the concentration - we deal with external economies of scale and scope;

(c) markets are imperfectly competitive; thus firms are endowed with some degree of market power - shaping the price above marginal costs because of the utilisation of internal economies of scale; with respect to these two possibilities exist:

- monopolistic competition - many companies, lack of strategic interaction, the product is diversified;

- partial oligopolistic competition (few actors with similar market power, strong strategic interactions, the existence of the Nash equilibrium game theory).

Combes, Mayer, and Thisse (2008) show that the choice of the above modelling strategy has significant implications for the results obtained. Approaches 1, 2, and 3a from the macro perspective bypass the role of individual companies. Approach $3 \mathrm{~b}$ considers strategic interactions between agents. The first approach generates a solution that is socially efficient in the sense of Pareto, and the remaining models lead to socially inefficient solutions. Combes et al. (2008) state that the classic NEG model chooses the third approach, where location decisions become endogenous at the expense of assuming the homogeneity of space (thus omitting the first nature of geography).

The basic model of the NEG of Krugman (1991a, 1991b) with a typical core-periphery structure (CP) is based on the concept of the monopolistic competition of Dixit and Stiglitz (1997). NEG models focus on the relationships between three factors determining location decisions of market agents: agglomeration benefits, non-zero costs of transport, and interregional migration (Fujita et al., 2001; Fujita and Thisse, 2004).

The cost of transport (resistance to overcome space) is primarily a function of the distance between trading parties but may also be a function of institutional barriers.

A key feature of the NEG models is the endogenization of the location decisions, and thus endogenization of location and distribution of economic activity in space (Brakman, Garretsen, and Schramm, 2004; Brülhart, 2001). Endogeneity of the location decisions means that market players and households consciously decide on the selection of their location in order to maximize profits (firms) or their total utility (consumers) based on the information (including prices) generated by the market system.

The market structure in the NEG models gradually evolves in the spatial domain under the influence of such factors as the size of the economy of individual regions; the costs of transport; the scope of internal economies of scale associated with the size of the production in individual production plants; the scope of the external economies of scale related to the degree of concentration of economic activities in the different sectors (the classic Marshallian externalities); the presence of linkages (ascending and descending or forward/backward linkages) in value-added chains; the significance of HME; the intensity of competition in a market is a function of the number of firms and their size-distribution (market size and economies of scale, competitive 
effect) as well as the interaction between them (e.g. the existence of restrictive collusion or exclusionary competition).

The degree of spatial concentration or dispersion of economic activity depends on the balance between centripetal (pro-agglomerative) and centrifugal (pro-dispersal) forces. It is worth noting that various NEG models differ in the direction of the effect and the significance of particular forces. For instance, external economies of scale are a natural pro-agglomeration force leading both to the creation of within-sector concentrations of firms (industrial districts or clusters) or multi-sectoral agglomerations of firms or households (cities).

In the standard NEG model with monopolistic competition, we deal with a differentiated product, homogeneous companies specialize in the production of its specific varieties, firms pose a certain degree of market power, and the nature of competitive rivalry is thus imperfect. At the same time, the number of enterprises in the market is large enough to get rid of the problem of potential strategic interactions characteristic for oligopolistic models. The framework is quite often criticized for oversimplification, and some authors try to take account of the strategic interactions in the location decisions of agents, for example using the Cournot oligopoly model (Combes and Lafourcade, 2011).

In NEG models we typically deal with multiple equilibria of unstable or stable character (as evidenced by the so-called tomahawk diagrams). Several corner solutions are possible:

(a) the total concentration of production activities in one of the two regions concerned (catastrophic agglomeration);

(b) total dispersion of production activities (the symmetric layout, equal shares of both territorial units);

(c) intermediate asymmetric equilibria of typically unstable character.

Furthermore, in NEG models, short- and long-term equilibria can be distinguished. The long-term allows for structural changes in the economy and thus adjustment in the location of economic agents.

An important role in regional development processes plays a level of mutual openness (in other words, the degree of integration of the regional economy) which is by definition linked to the level of transport costs. The transport costs are typically modelled as the so-called ice-berg transport costs (Samuelson, 1954) in which transport costs linearly relate to distance, and they work by extracting from the arriving volume.

Transport costs reflect the specific resistance of space, leading to concentration or dispersion of production. Recent studies have proven the relationship between the level of transport costs and the degree of concentration of business to be nonmonotonic and nonlinear having the shape of an inverted U (Ottaviano, 2008). High transport costs lead to the dispersion of economic activity. As transport costs fall to 
medium levels, we observed gradual concentration. If they fall further to low levels, dispersion of economic activity emerges once again.

The lowering of the cost of the exchange is the result of among others:

(a) technological progress through the use of more efficient means of transport, better organisation of logistic processes, etc.;

(b) utilisation of economies of scale in transport that leads to a decrease in the unit costs;

(c) improvement in the transport infrastructure leading to increased inaccessibility of the regions;

(d) reduction in the level of protection of the relevant market, and thus an increase in the level of mutual openness of the economies in the region.

In this framework, one can explore the impact of integration (asymmetric reduction in trade costs) on the location of economic activity. For example, Hanson (1994) examined the effects of a fundamental change of the trade policy regime in Mexico from protectionist to liberal in 1984, on the location of the processing industry within the country. In a relatively short time from trade liberalisation, it led to a significant contraction of the prior concentration of manufacturing industry around Mexico City (the so-called Mexico City manufacturing belt) which initial emergence was related to the implementation of the costly policy of import substitution. Liberalisation resulted in the relocation of production plants to the regions directly on the border with the US. We thus deal with interregional adjustment in location and production/export capacity within a single economy as a result of the shock - trade liberalisation.

The results of empirical studies indicate that the integration processes within the framework of the EU also affect the location of business activity - leading to an increase in the degree of spatial concentration of production activities (Amiti, 1999; Brülhart, 2011; Midelfart-Knarvik and Overman, 2002). The flow between the core and peripheral regions of the EU depends on the specific circumstances of each sector (including the intensity of economies of scale, dependence on transport costs, and the intensity of the backward and forward linkages) and we observe heterogeneity in this area. Furthermore, each new enlargement leads to an adjustment in the location decisions of firms and an adjustment in the structure of individual markets due to mergers and acquisitions. Brülhart (2001) believes that at the aggregated level, the core-peripheries system, characteristic for the European Union, seems to fade away gradually. Midelfart-Knarvik and Overman (2002) state that the equalisation-oriented structural policy of the EU has a significant impact on the location of economic activity, not necessarily leading to the efficiency of the block taken as a whole.

Models of NEG, aiming to resolve the problem of the analytical burden, typically assume the existence of two regions and two sectors $(2 \times 2)$. In reality, both the number of regions and sectors is significantly higher with multi-region and multisector framework. In reality, we are also dealing with the subset of multiproduct and multiplant firms (with dispersed activities). 
Behrens, Lamorgese, Ottaviano, and Tabuchi (2004) point out that in the multiregional system even the static location of companies is determined by mutually reinforcing spatial effects (accessibility) and non-spatial effects (attraction), which affects the overall distribution of demand in all the regions concerned (third-country/ party effect). The economic potential of a specific region is a function of not only the size of the region but also its relative accessibility within the framework of the multiregional system (hence the popularity of the market potential approach).

However, even the most advanced models of spatial equilibrium, for instance for France (Combes and Lafourcade, 2001, 2011), taking into account the problem of strategic interaction (Cournot competition) in the location decisions of agents, or Teixeira (2006) for Portugal, have a static character and thus ignore the problem of growth dynamics. NEG models trying to explain the evolution of the location of activities in space simply overlook the problem of the economic growth process.

Combes and Lafourcade (2011) state that they cannot tell whether France is close to a short- or long-run spatial equilibrium. They note that from a regional perspective, France is gradually moving from the system with one core (Ile de France - Paris) to a system with two cores - the other one emerging in the south-eastern part of the country.

These complex scenarios, including endogenous location choices and endogenous growth, are only possible in models combing static NEG with postulates or mechanisms characteristic for the previously described new growth theory, the socalled dynamic NEG models. This subset of models includes Fujita and Thisse (2006), Martin and Ottaviano (1996) or Baldwin and Forslid (1999; 2001).

Dynamic NEG models with endogenous growth assign a significant role to externalities arising from human capital accumulation or the broader concepts of knowledge. The externalities' effects are decreasing in the distance, which means that they are localized (Hanson, 2001). The same factors that determine the location of economic activity in the context of dynamic models of NEG are the same ones that are responsible for the endogenization of economic growth.

Baldwin et al. (2011) distinguish three types of dynamic NEG models. These are core-periphery models (CP); footloose entrepreneur models (FE); footloose capital models (FC).

The standard NEG model in the Dixit-Stiglitz-Krugman formula includes the following structure and set of assumptions:

(a) two regions - developed North (N) and the underdeveloped South (S);

(b) two sectors of the economy - agriculture (A) and the manufacturing industry (M);

(c) two factors of production - physical capital (K) and labour (L);

(d) one of the factors of production is mobile, and the second is immobile;

(e) A sector - Walrasian agricultural sector with perfect competition producing a homogeneous product with constant economies of scale; 
(f) $\mathrm{M}$ sector - manufacturing sector producing $\mathrm{n}$ varieties of a differentiated product in the presence of increasing economies of scale within monopolistically competitive market;

(g) each of the varieties of the differentiated good is produced by another company which within this given variety possess market power - a monopolist rent, and thus sets prices above of marginal costs;

(h) firms are however homogeneous (representative firm model) or symmetric;

(i) preferences of firms and households are homogeneous;

(j) regions share the same level of technological sophistication.

A typical mainstream NEG model, a la Krugman (1991a, 1991b), is characterized by the following features:

(a) circular causality on the demand side (which offset production moves) and on the supply side (production costs, offset production affects the level of costs);

(b) hysteresis in the location of economic activity, that is, the present distribution in space depends on the preceding situation (path-dependency);

(c) the occurrence of multiple stable or unstable equilibria;

(d) the presence of symmetric ( $\mathrm{N}=\mathrm{S}$, dispersed equilibrium) as well as severely unbalanced equilibria (the tomahawk diagram);

(e) the bell-shaped chart depicting the evolution of regional development;

(f) the presence of catastrophic agglomeration due to the homogeneity of preferences;

(g) the presence of HME;

(h) the existence of the so-called spatial structure of wages reflecting the variation in productivity;

(i) non-linearity (non-monotonic character) of many associations, such as the impact of transport costs on the level of the spatial concentration of economic activities.

In dynamic models, in contrast to static approaches, processes have a temporal dimension, which creates a fundamental difference. In terms of the medium-term, changes (shifts) in economic policy result is a one-time adjustment of capital, which is the flow and allocation of capital between regions, although the rate of accumulation is unchanged - we thus deal with the level effect. In the long-run, economic policy changes can affect the pace of capital accumulation, and thus we deal with the rate of a growth effect.

Effects for the overall level of prosperity in the case of models with a fixed level of capital (K) are as follows:

(a) the effect of prices on the border - the decrease in import prices rises prices in exports which causes an increase in the prosperity of the region $\mathrm{N}$ according to the classic definition of the terms of trade effect; 
(b) the effect of location on the cost of living - if the number of varieties of differentiated good $n$ is constant, the dislocation of the production of one variety of the differentiated good from the region $\mathrm{S}$ to $\mathrm{N}$ causes an increase in the prosperity of the region; the significance of the effect increases with the increase in transportation costs;

(c) the effect of migration depends on the type of model, in CP and FE models an inflow of mobile capital $\mathrm{K}$ raises real income; in the FC model, it has no impact on the level of prosperity.

In the case of dynamic models with capital accumulation, the capital accumulation occurs until the steady-state level is reached, for which the value of an additional unit of capital aligns with the cost of its production. In the steady-state, further accumulation of capital bring zero effects for the general welfare.

Baldwin et al. (2001) distinguish two NEG models with an accumulation of capital: (a) the constructed capital (CC) model (Baldwin, 1999); (b) localized (LS) or global (GS) spillover models.

In the CC model, with the accumulation of capital, each new unit of capital is associated with the emergence of a new variety of differentiated goods. The increase in the number of available varieties is thus the manifestation of technological progress, by analogy with the horizontal differentiation models of endogenous growth literature. The process of accumulation of capital thus translates into an increase in several available varieties. It causes a progressive decline in the general level of prices, and therefore a gradual increase in the real product and real wages.

LS and GS models also allow for the endogenization of economic growth. The key feature is the adoption of a broad definition of capital - physical capital, human capital, and knowledge considered together bypasses the problem of diminishing returns through the incorporation of learning curve effects in the production process. Thus, the cost of broadly defined capital decreases in time. Capital of this type may spill into adjacent areas (imperfect diffusion, LS - localized spillovers) or globally (perfect diffusion, GS - globalized spillovers).

Knowledge diffuses primarily within individual economic sectors (intra-sectoral spillovers, the so-called MAR externalities) and to a more limited extent between sectors (inter-sectoral spillovers, or the Jacobian externalities). The localized spillovers are the main forces responsible for the spatial concentration of sectors or overall spatial agglomeration of economic activity and population, explaining the phenomena of industrial districts, clusters, and the emergence and development of cities and metropolitan centres.

In the LS models, we are dealing with a perfect diffusion of knowledge between enterprises in a given region (for example, within a single cluster) and hindered interregional knowledge diffusion. The scale of localized diffusion location choice has an impact on the long-term growth rate. 
In the GS models, knowledge defuses between firms from different regions. In this setting, capital accumulation can lead to catastrophic agglomeration. Spatial considerations do not affect the long-term growth rate; however, they have an impact on the level of development (through transitional effects).

In the LS and GS models, private atomistic innovators overlook the presence of externalities in their activities and thus the impact of their activities on the evolution of the general price levels. At the same time, the learning effect occurs in the innovation sector. The apparent market failure leads to the socially suboptimal growth rate in the Pareto sense and thus creates an opportunity for a potentially favourable public intervention (e.g. R\&D subsidy). The dynamic NEG models with knowledge spillovers include models of Martin and Ottaviano (1996). Baldwin and Forslid (1999), Baldwin et al. (2001) Baldwin and Martin (2004).

Endogenization of growth in the NEG model requires similarly to models of the new growth theories, the extension of the concept of capital and taking into account externalities in its accumulation, or endogenization of the technological progress by the introduction to the structure of the model of an R\&D sector responsible for the creation of new knowledge and innovation. In the process of knowledge generation, knowledge diffuses to the neighbouring regions.

From the theoretical point of view, diffusion of knowledge can be perfect (global and immediate diffusion) or imperfect (spatially restricted or localized). Numerous empirical results confirm that the diffusion of knowledge is imperfect and is strongly localized, despite the progress in the field of information technology (Ciccone, 1996; Coe et al., 1997; Eaton and Kortum, 2001; Jacobs, 1970; Jaffe, Trajtenberg, and Henderson, 1993; Keller, 2002, 2004; Thompson and Fox-Kean, 2005; Zucker et al., 1998). The recent evidence for Poland also points to the localized character of knowledge diffusion (Ciołek and Brodzicki, 2016, 2017). The localized knowledge spillovers are in brief due to the tacit character knowledge which diffusion requires direct face-to-face interaction.

Dynamic NEG models are far from ideal. They are foremost, not able to fully capture the complex nature of space or the interactions between actors (Zaucha, 2008). To some extent, they represent a compromise between the desire to capture as much of the spectrum of conditions and factors and the objective limitations on the modelling side.

NEG models cannot be considered as exhaustive coverage of spatial issues in the analysis of economic growth. They are excessively one-sided and respond to the question on the role of space in the process of economic growth, refer only to the cost of tackling space. In this situation, spatial concentration is the factor, which promotes development. The alternative approach is the postulate of space without the cost of its overcoming. The NEG, however, omits the costs of "density", which may negatively impact the growth rate of GDP through congestion (Zaucha, 2007, 2008).

Fujita and Krugman (2003) stated that future models must have even richer microeconomic foundations, rely to a greater extent on the results obtained through 
empirical analyses, and relate directly to the consequences for the well-being of individual societies, and therefore, generate better recommendations for economic policy.

The newest theoretical models a la Melitz (2003) move away from the traditional assumption of firm homogeneity (representative company models) and consider the actual heterogeneity of firms in terms of, e.g. productivity, size, or scope of activities. These models, taking into account the stochastic distribution of productivity, lead to meaningful and new theoretical postulates, for example on the cause of the occurrence of the exporters, competitiveness, internationalisation, and innovation, but at the same time lose the simplicity and thus transparency characteristic for former models and thus lead to unambiguous economic policy recommendations.

Ottaviano (2011) notes that future NEG models should account for both macroheterogeneity across locations and micro-heterogeneity across firms within sectors and people with various preferences. Taking the firm heterogeneity into consideration leads to the emergence of the next generation of models - the so-called new NEG (NNEG) models. Ottaviano states: "still based on the pillars of scale economies and imperfect competition but with a stronger emphasis on how individual heterogeneity across people and firms maps into aggregate behaviour”.

For instance, Baldwin and Okubo (2006) built a model of the NNEG-type integrating a heterogeneous firm's Melitz-style model of monopolistic competition with a simple NEG model obtaining several interesting results. First, only the most productive firms can benefit from reallocation to larger regions. The selection effect exists, decreasing the extent of traditional agglomeration economies. Furthermore, a spatial sorting effect arises that induces the highest productivity firms to move to the core and the lowest productivity firms to the periphery. Furthermore, the HME is weaker due to firm heterogeneity. According to Ottaviano (2011), in the model firm heterogeneity acts as an additional centrifugal force - the greater, the larger are the trade costs and the larger the substitutability between firms' products.

Forslid and Okubo (2010) extended the Baldwin and Okubo (2006) model by introducing different capital intensities among firms (that can move between regions) and sectors. More productive firms were assumed to be more capital intensive. As a result, the model postulates sorting to the large regions from both ends of the actual productivity distribution (Pareto or log-normal distribution of TFP is observed in the actual firm-level data). Specifically, firms with high capital intensity and high productivity as well as firms with very low productivity and low capital intensity tend to relocate to the core (core region premium). Using Japanese data, authors provide some evidence for the predicted two-sided sorting and in particular in high capital intensity sectors. The early NNEG models include as well the works by Baldwin and Okubo (2009), Nocke (2006) or Okubo (2009).

In a recent paper, Forslid and Okubo (2019) proposed an NNEG model incorporating firm heterogeneity in which multiproduct firms can relocate between regional markets (core and periphery). In the framework, the product scope of a firm increases in the market size and simultaneously, the product scope of a firm increases in its 
productivity. The most productive firm has, at the same time, the highest incentive to deviate to the core region. Trade liberalisation in this framework thus leads to relocation of the most productive firms to the larger market (core region), where they further expand their product scope (given the relocation costs). The less productive firms with a smaller product range located in the smaller market contract their product range due to increased competition. The above effects are magnified with the productivity of the migrating firm. The pro-agglomerative effect is, however, weakened as only the most productive firms with the largest product scope can locate in or migrate to the core region. Forslid and Okubo (2019) find the above theoretical postulates to be consistent with the data on location and product scope of manufacturing firms located in Japanese prefectures.

Brülhart (2011) in the conclusions of his survey of implications of trade liberalisation for intra-national geographies of individual economies (intra-national regional inequality) states that the results of both urban systems and NEG models are inconclusive. Everything depends on the modelling strategies and choices adopted. Empirical results are rather inconclusive as well; however, a majority of cross-country studies find no significant effect of openness on urban concentration or regional inequality. Whether trade liberalisation (or greater openness to trade) raises or lowers regional inequality depends on country-specific geography, which is to a large extent of exogenous character. The greatest benefits are likely to emerge in the case of regions, ceteris paribus, with inherently less costly access to foreign markets - border or port regions.

González Rivas (2007) postulates that an ability of a given region to capture the benefits related to greater trade openness depends primarily on critical endowments and thus the degree to which trade is likely to reduce regional inequality in a given country is mediated by the geographic distribution of its endowments. He tested the hypothesis for Mexico on a sub-national dataset from 1940. The results indicate that liberalisation benefits to a greater extent of regions with lower levels of education, thereby tending to reduce regional inequality. However, it also benefits more regions with higher levels of income and infrastructure, thereby tending to increase regional inequality. The second effect is stronger; thus, trade openness increases regional inequality.

Krugman (2010) states that recent developments in the regional evolution of China are in line with the postulates of the standard core-periphery model that predicts increasing regional specialisation as a result of economic integration (liberalisation).

Redding (2010) points to a major weakness of NEG theory in the empirical domain. Earlier Krugman (1998) noting the theoretical contribution of NEG by incorporating space into mainstream economics, fully inspecting the impact of increasing returns and identifying many non-linear aspects, stated that the weakness lied in lack of convincing empirical verifications. Nonetheless, some of the analyses - for instance by Davis and Weinstein (1996, 1998; 2002; 2008) - were very encouraging; however, they pointed to some important problems such as the assumption of homogeneity of preference (not in line with the rebuilding of Nagasaki or Hiroshima after the Second 
World War). On the other hand, Davis and Weinstein (2003) proved the existence of the home market effects for a broad segment of the OECD manufacturing industry.

It is worth pointing out that economic geographers frequently question the basic assumptions, the structure, and logic of NEG models. Martin (1999a) considers it just like a reworking of traditional location theory and regional science using recent developments in formal (mathematical) mainstream economics. Therefore, they also question the policy relevance of this kind of modelling (e.g. Martin and Sunley, 2011).

Krugman (2010) on the other hand thinks that the possibilities of convergence between economic geography and NEG are rather dim by stating "Although both economists and geographers study these spatial processes, no fruitful exchange between the two is expected because of the use of different methodologies".

Garretsen and Martin (2010) point to two important weaknesses of NEG models: the oversimplified treatment of geography (pre-given, fixed and highly idealized abstract geometric space) and history (logical time and not real history). One of the first steps in this process is made in an empirical paper by Bosker et al. (2010) where the authors move from unidimensional NEG model and propose a strategy combining estimation and simulation accounting for heterogeneous and complex geographical structures. A combination of the estimation of structural NEG parameters with a simulation of the underlying multidimensional NEG model more accurately links the empirical results to the theory.

Storper (2011) postulates that only big changes in the openness to trade (for instance adjustments in the tariff levels or establishment or abolishment of trade barriers or trade liberalisation schemes) considered as major shocks to an economic system may result in significant structural adjustments in firms' decisions resulting in their reorganisation and potentially re-location as long as the shocks are big enough to overcome existing sunk costs and/or agglomeration economies and then readjustments in the location of labour.

Yu, Zhao, and Ming (2006) analysed the causes of industry agglomeration in China at the provincial level of spatial disaggregation over the period 1987-2001 using the NEG modelling framework. The authors conclude that liberalisation led to industry agglomeration. Furthermore, factors such as market size, the level of urbanisation, or investments in the infrastructure promoted industrial agglomeration. Coastal Chinese regions at the same time enjoy clear geographical advantages promoting their accelerated industrial growth.

Summing up, the bond between economic growth and openness of regions is complex and to some extent, an ambiguous issue. The relationship becomes even more blurred if we take into account the nonlinearity and heterogeneity of space. Nonetheless, overlooking the spatial interactions at the regional level of analysis, and thus of NEG or NNEG postulates, could lead to falsified conclusions and thus to wrong policy recommendations. 